\title{
Prior Bariatric Surgery is Associated with a Reduced Risk of Poor Outcomes in COVID-19: Propensity Matched Analysis of a Large Multi-institutional Research Network
}

\author{
Yousaf Bashir Hadi ${ }^{1}$ Rupinder Mann ${ }^{2} \cdot$ Amir Humza Sohail $^{3} \cdot$ Miles Graves $^{4} \cdot$ Nova Szoka $^{5} \cdot$ Salim Abunnaja ${ }^{5}$. \\ Lawrence E. Tabone ${ }^{5} \cdot$ Shyam Thakkar ${ }^{1} \cdot$ Shailendra Singh ${ }^{1}$ (i)
}

Received: 29 July 2021 / Revised: 6 November 2021 / Accepted: 10 November 2021 / Published online: 23 November 2021

(c) The Author(s), under exclusive licence to Springer Science+Business Media, LLC, part of Springer Nature 2021

\begin{abstract}
Purpose Through sustained weight loss and improvement in metabolic co-morbidities, bariatric surgery is hypothesized to reduce the risk of severe COVID-19. Small studies have suggested favorable outcomes; however, large population-based studies are lacking.

Materials and Methods We conducted a retrospective cohort study utilizing the multi-institutional research network TriNeTx platform. Participants diagnosed with COVID-19 were identified and divided into cohorts based on prior bariatric surgery (BS). Primary study outcome was a composite event of death or requirement for mechanical ventilation up to 30-day following the diagnosis of COVID-19. Other outcomes included death, hospitalization, critical care need, and acute kidney injury in the 30-day follow-up period. Outcomes were compared in BS and non-BS cohorts after propensity score matching. Results There were significant differences in patient demographics and co-morbidities between the BS and non-BS groups. In the propensity score-matched analysis, there was a lower risk of reaching the primary endpoint of mechanical ventilation or mortality at 30 days after COVID-19 diagnosis in the BS cohort compared to the non-BS cohort (risk ratio (RR) 0.40 , 95\% CI 0.25-0.65). Mortality rate was lower in the BS cohort (RR 0.42, 95\% CI 0.22-0.80), and patients in the BS group were less likely to require critical care (RR $0.54,95 \%$ CI $0.38-0.77$ ), mechanical ventilation (RR 0.43 , 95\% CI $0.24-0.78$ ) or develop acute kidney injury (RR $0.57,95 \%$ CI $0.43-0.76$ ) after COVID-19 diagnosis.

Conclusion Prior bariatric surgery is associated with a reduced risk of poor outcomes of COVID-19. Furthermore, large prospective studies are needed.
\end{abstract}

Keywords COVID-19 $\cdot$ Bariatric surgery $\cdot$ RYGB $\cdot$ Gastric bypass $\cdot$ Sleeve gastrectomy

Key Points:

- Lower mechanical ventilation rate and mortality were seen after COVID-19 diagnosis in patients with prior bariatric surgery compared to patients without a history of bariatric surgery. - Overall, good outcomes of COVID-19 disease in patients with a history of bariatric surgery were observed.

- No differences in outcomes of COVID-19 were noted between patients with prior RYGB and sleeve gastrectomy.

Shailendra Singh

Shai1121@gmail.com

Extended author information available on the last page of the article

\section{Introduction}

SARS-CoV-2-related COVID-19 pandemic has emerged as a major global health crisis. Co-morbid illnesses including heart disease, chronic lung disease, diabetes mellitus, hypertension, and increasing age have been identified as main risk factors for severe clinical course of this illness [1]. Previous data has also pointed towards an increased risk of worse outcomes with COVID-19 in patients with obesity. The Lille Intensive Care COVID-19 and obesity study group reported an increased risk of mechanical ventilation in patients with high BMI [2]. More than three-fold higher risk of critical care need was noted in young patients with body mass index $>35 \mathrm{~kg} / \mathrm{m}^{2}$ admitted with COVID 19 in a report from New York City [3]. Several studies have established obesity as an independent risk factor for severe COVID-19 
disease course $[4,5]$. Obesity may increase the risk of severe disease both by its associated metabolic and cardiovascular diseases, and by alterations in respiratory mechanics, and increase in risk of thrombotic phenomenon. Furthermore, increased secretion of cytokines in excess adipose tissue and persistent low-grade inflammation associated with obesity may contribute to immune dysfunction in the setting of COVID-19 disease [6].

Bariatric surgery is commonly performed for treatment of obesity, with Roux-en-Y gastric bypass (RYGB) and sleeve gastrectomy (SG) being the most frequently performed bariatric procedures in the Western world [7]. Through sustained weight loss and improvement in metabolic co-morbidities, a history of bariatric surgery may be hypothesized to reduce the risk of severe COVID-19 disease. Furthermore, the ACE-2 enzyme to which SARS-CoV-2 binds is expressed substantially in the gastric mucosa [8], and thus bypass or exclusion of stomach can be speculated to alter the course of COVID-19 in a mechanism independent of obesity. A substantial number of individuals have a history of these bariatric procedures; however, the outcomes of COVID-19 disease in this population have not been sufficiently explored. Some preliminary data point towards favorable outcomes of COVID-19 in patients with bariatric surgeries, however, due to small sample size and lack of robust control, definitive conclusions cannot be drawn $[9,10]$. Therefore, we conducted a propensity-matched analysis of a large multi-institutional research network to study the outcomes of COVID-19 disease in patients with a history of bariatric surgery.

\section{Methods}

\section{Study Design and Description of Data Source}

We conducted a retrospective cohort study utilizing the multi-institutional research network TriNetX (Cambridge, MA, USA) platform. TriNetX is a federated multi-institutional research network including more than 45 Health Care Organizations (HCOs) in the USA that provides real-time access to healthcare records of more than 40 million patients from the included HCOs. The included data is directly retrieved and aggregated from the electronic health record systems (EHRs) of participating organizations in real-time. The organizations included in the TriNetX network are mostly large academic centers operating both tertiary care and satellite outpatient office locations.

Clinical variables (referred to as "facts" on the network) are derived directly through EHRs. Robust quality assurance on the network is achieved at the time of extraction before inclusion. The platform only provides aggregate patient counts and statistical summaries to ensure de-identification at all levels of retrieval and dissemination of patient data. TriNetX has received a waiver from Western IRB as a federated research network as the platform only provides de-identified information. Details of TriNetX network can be found at https://trinetx.com/\# covid and are described in previous studies $[11,12]$.

\section{Study Participants}

Patients over 16 years of age diagnosed with COVID-19 were identified using search methodology based on CDC and TriNetX COVID-19 coding guidance. International Classification of Diseases, Ninth Revision and tenth Revision, Clinical Modification (ICD-10-CM) codes and Logical Observation Identifiers Names and Codes (LOINC) codes for positive laboratory tests were used. This search strategy was previously used in published data from the TriNetX [11, 12]. These codes are detailed in Supplementary Table 1. Patients diagnosed with COVID-19 were stratified into two cohorts.

a. Participants with a history of bariatric surgery at least two weeks before the diagnosis of COVID-19 were identified and included in the bariatric surgery (BS) group. Bariatric procedures included gastric bypass, sleeve gastrectomy, and laparoscopic gastric band placement.

b. Patients with no history of bariatric procedures who were diagnosed with COVID-19 were identified and included in non-bariatric surgery control (non-BS) group.

Selection methodology is detailed in the supplementary file.

\section{Study Duration}

A real-time search and retrospective analysis was conducted on the TriNetX platform. Diagnosis of COVID-19 between January 20, 2020 and April 30, 2021 was required for inclusion in the study cohort. January 20, 2020 was chosen as it was the date of diagnosis of the first case of SARS-CoV-2 infection in the USA. April 30, 2021 was selected to ensure at least 90 days of follow-up for all included patients.

\section{Study Definitions and Outcomes}

Index event was defined as date of first positive SARSCoV-2 test or COVID-19 diagnosis. Study outcomes were assessed at 30 days from index event and included the day of index event. Baseline characteristics for patients were considered until the day of COVID-19 diagnosis or first positive SARS-CoV-2 test result. 
Primary study outcome was a composite of death or requirement for mechanical ventilation at 30-day from the index event. Other outcomes included mortality, hospitalization, critical care need, and acute kidney injury in the 30-day follow-up period. Composite outcome and mortality were also estimated at a 90-day follow-up period.

\section{Statistical Analysis}

TriNetX platform was used to conduct all analyses. For the primary analysis, study outcomes were compared between the BS and Non-BS cohorts. Chi-square tests and T-tests were used for univariate analysis. A 1:1 matching was performed based on the propensity scores generated by using greedy nearest neighbor algorithms utilizing a caliper width of 0.1 pooled standard deviations (SD). TriNetX randomizes the order of rows in order to eliminate bias resulting from nearest neighbor algorithms. Propensity score matching was performed with age, gender, race, body mass index (BMI), diabetes, hypertension, chronic lung diseases, nicotine dependence, heart failure, and ischemic heart disease as covariates. Balance on covariates was assessed using standardized mean difference, and absolute values $>0.1$ were considered positive for residual imbalance. A two-sided alpha of less than 0.05 was defined a priori for statistical significance. Risk ratios with $95 \%$ confidence intervals were calculated for all analyses. TriNetX obfuscates patient counts when aggregate count $\leq 10$ to safeguard protected health information. Survival analysis was performed by plotting KaplanMaier curves with log rank tests to compare survival in the BS and non-BS cohorts.

Matched and unmatched subgroup analysis was performed to compare outcomes between RYGB and SG groups. Matching was performed for the same covariates mentioned above (age, race, gender, BMI, diabetes, hypertension, chronic lung diseases, nicotine dependence, heart failure, and ischemic heart disease).

\section{Sensitivity Analysis}

Sensitivity analysis was performed by comparing outcomes between the BS cohort and the 'bariatric procedure qualifying' cohort. Patients with no history of bariatric surgery but with clinical attributes that would qualify for these procedures were identified and included in the 'bariatric procedure qualifying' group ( $\mathrm{BMI} \geq 40$ or if they had $\mathrm{BMI} \geq 35$ with the presence of a co-morbid condition including hypertension, diabetes mellites type 2 , hyperlipidemia, ischemic heart disease or heart failure, or obstructive sleep apnea). After unmatched crude analysis, two propensity scorematched analyses were performed. Propensity scorematched analysis was first performed by matching propensity scores incorporating demographic variables and lower respiratory disease only, with no matching performed for metabolic diseases and BMI, to prevent bias resulting from inadvertent control of effects of bariatric surgery. Subsequently, a matched analysis was performed between these cohorts with all the abovementioned covariates (age, race, gender, BMI, diabetes, hypertension, chronic lung diseases, nicotine dependence, heart failure, and ischemic heart disease).

\section{Results}

\section{Study Population}

Of the patients who were identified to have COVID-19 by inclusion criteria, 1940 patients had a history of bariatric surgery and were included in the BS cohort. The 979,981 participants did not have a history of bariatric surgery and were included in the control (non-BS) group. Of the bariatric surgery cohort, 887 and 952 patients were included in the RYGB and SG groups, respectively, while the rest of the patients had a history of gastric banding. A cohort of 72,521 patients was identified with no history of bariatric procedures who fulfilled criteria for "Bariatric surgery qualifying" group.

\section{Baseline Characteristics of Study Cohorts}

At the time of COVID-19 diagnosis, mean age in the bariatric surgery group was 48.13 years (SD 11.89 years). Among the bariatric surgery cohort, a majority of participants were female $(82.32 \%)$. White race was most common $(63.25 \%)$. Geographically, in the BS cohort, $37 \%$ of patients were from southern USA, $25 \%$ from the Northeast, while Midwest and Western US contributed $30 \%$ and $7 \%$ patients, respectively.

There were significant differences in demographics and co-morbidities between the BS and non-BS groups. Female gender was more common in BS cohort compared to non-BS cohort $(p<0.01)$. BS patients had higher mean age, Caucasian race, obesity, hypertension, diabetes, chronic kidney disease, nicotine dependence, heart failure, chronic liver disease, and ischemic heart disease compared to non-BS cohort at baseline (all $p$ values $<0.05$ ) (Table 1$)$.

After COVID-19 diagnosis, $22.27 \%$ of patients received steroids in the BS group compared to $23.6 \%$ in the nonBS group (RR 0.94, 95\% CI 0.84-1.06). Two thousand and two-hundred thirty patients $(0.23 \%)$ in non-BS group had received vaccination before COVID-19 diagnosis, while $\leq 10$ $(\leq 0.51 \%)$ participants in the BS group were vaccinated against COVID-19 at the time of COVID-19 diagnosis. 


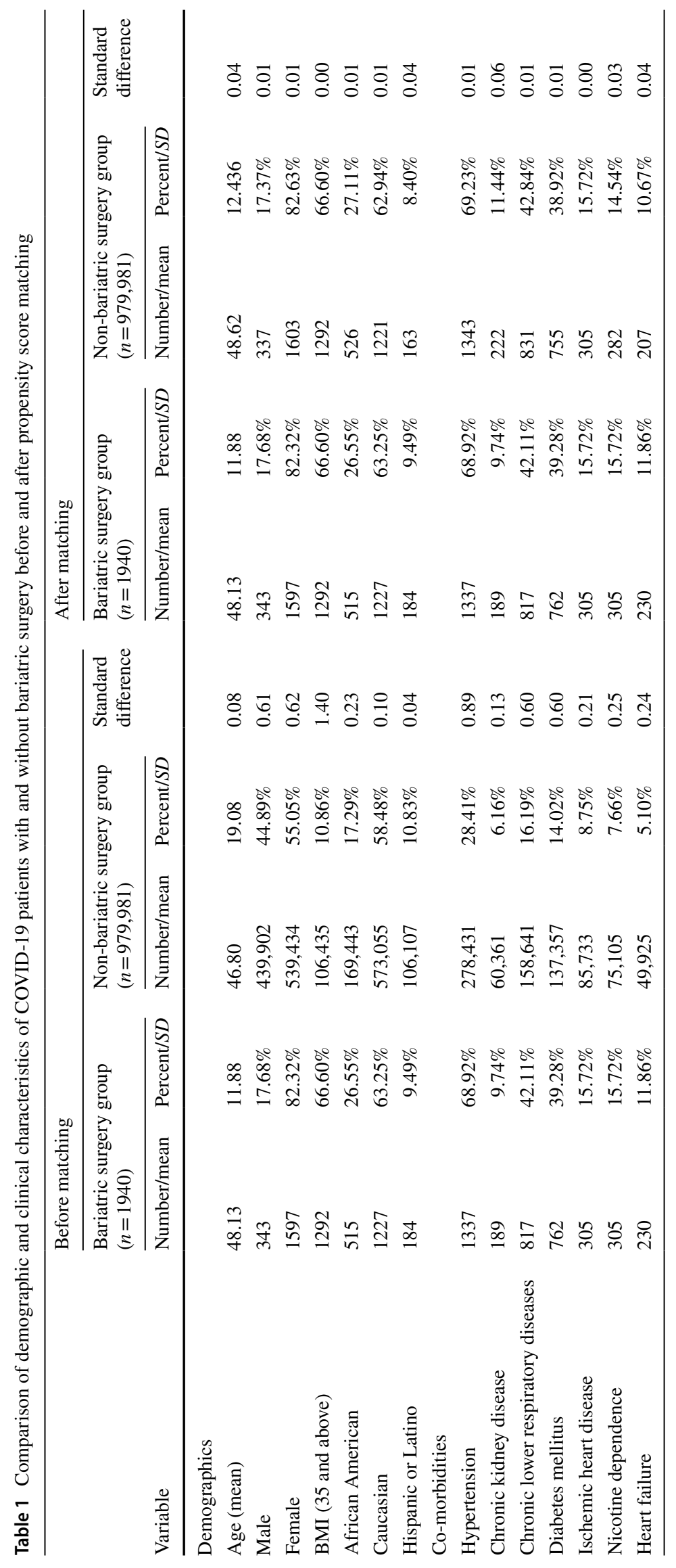




\section{Outcomes}

\section{COVID-19 Disease Outcomes in Bariatric Surgery Cohort}

In the BS group, 193 (10.25\%) patients required hospitalization, and 15 patients $(0.77 \%)$ required mechanical ventilation in the 30-day follow-up period after COVID diagnosis. Critical care services were required by 45 patients $(2.32 \%)$ in the 30-day follow-up period. Twenty-three $(1.19 \%)$ and $26(1.34 \%)$ patients in the BS group reached the primary composite endpoint of death or mechanical ventilation in the 30-day and 90-day follow-up, respectively.

\section{Comparison with Non-bariatric Surgery Cohort}

Crude unmatched analysis revealed a lower risk of reaching primary composite endpoint of mechanical ventilation or mortality at 30 days and 90 days after COVID19 diagnosis in the BS cohort compared to the non-BS cohort. Lower risk of need for mechanical ventilation and lower mortality was noted in the bariatric surgery cohort (Table 2).

After propensity score matching, baseline clinical characteristics in the two groups were similar, and no residual imbalance was found (standard difference $<0.1$ for all covariates). Baseline characteristics of the matched and unmatched groups are shown in Table 1.

In the propensity score-matched analysis, there was lower risk of reaching the primary endpoint of mechanical ventilation or mortality at 30 days after COVID-19 diagnosis in the BS cohort compared to the non-BS cohort (1.19\% vs. $2.94 \%$, risk ratio $0.40,95 \%$ CI $0.25-0.65$ ). Lower risk of reaching the primary composite endpoint of mechanical ventilation or mortality at 90 days after COVID-19 diagnosis was also noted in the BS cohort $(1.34 \%$ vs $3.04 \%$, risk ratio $0.44,95 \%$ CI $0.28-0.70$ ). Mortality rate was lower in the BS cohort, and patients in the BS group were less likely to require critical care, mechanical ventilation, or develop acute kidney injury after COVID-19 diagnosis (Table 2).

No difference was noted in laboratory values of C-reactive protein, bilirubin, ALT, AST, ferritin, bilirubin, and lactate dehydrogenase after COVID-19 diagnosis between the study cohorts on both matched and unmatched analysis; however, mean ferritin level was higher in the non-BS cohort (Table 3).

\section{Survival Analysis}

Kaplan-Maier survival curves were plotted for mortality and composite outcome (death or mechanical ventilation) for

Table 2 Outcomes in the two cohorts of COVID-19 patients with history of bariatric surgery and without bariatric surgery before and after propensity score matching

\begin{tabular}{|c|c|c|c|c|c|c|c|}
\hline \multicolumn{8}{|c|}{ Before propensity score matching } \\
\hline Outcome & $\begin{array}{l}\text { Bariatric } \\
\text { surgery group } \\
(n=1940)\end{array}$ & Percentage & $\begin{array}{l}\text { Non-bariatric } \\
\text { surgery group } \\
(n=979,981)\end{array}$ & Percentage & Risk ratio & $95 \% \mathrm{CI}$ lower & 95\% CI upper \\
\hline Mortality within 30 days & 13 & 0.67 & 17,388 & 1.77 & 0.38 & 0.22 & 0.65 \\
\hline Mortality within 90 days & 17 & 0.88 & 21,684 & 2.21 & 0.39 & 0.25 & 0.64 \\
\hline Inpatient services & 199 & 10.25 & 69,565 & 7.10 & 1.44 & 1.27 & 1.65 \\
\hline Critical care & 45 & 2.32 & 24,307 & 2.48 & 0.94 & 0.7 & 1.25 \\
\hline Mechanical ventilation & 15 & 0.77 & 14,080 & 1.44 & 0.54 & 0.33 & 0.89 \\
\hline 30-day composite outcome & 23 & 1.19 & 25,434 & 2.60 & 0.46 & 0.30 & 0.69 \\
\hline 90-day composite outcome & 26 & 1.34 & 28,986 & 2.96 & 0.45 & 0.31 & 0.66 \\
\hline Acute kidney injury & 64 & 3.30 & 37,403 & 3.82 & 0.86 & 0.68 & 1.1 \\
\hline \multicolumn{8}{|c|}{ After propensity score matching } \\
\hline Outcome & $\begin{array}{l}\text { Bariatric sur- } \\
\text { gery group } \\
(n=1940)\end{array}$ & Percentage & $\begin{array}{l}\text { Non-bariatric } \\
\text { surgery group } \\
(n=1940)\end{array}$ & Percentage & Risk ratio & $95 \%$ CI lower & 95\% CI upper \\
\hline Mortality within 30 days & 13 & 0.67 & 31 & 1.60 & 0.42 & 0.22 & 0.80 \\
\hline Mortality within 90 days & 17 & 0.87 & 41 & 2.11 & 0.41 & 0.24 & 0.73 \\
\hline Inpatient services & 199 & 10.26 & 210 & 10.83 & 0.95 & 0.79 & 1.16 \\
\hline Critical care & 45 & 2.32 & 84 & 4.33 & 0.54 & 0.38 & 0.77 \\
\hline Mechanical ventilation & 15 & 0.77 & 35 & 1.80 & 0.43 & 0.24 & 0.78 \\
\hline 30-day composite outcome & 23 & 1.19 & 57 & 2.94 & 0.40 & 0.25 & 0.65 \\
\hline 90-day composite outcome & 26 & 1.34 & 59 & 3.04 & 0.44 & 0.28 & 0.70 \\
\hline Acute kidney injury & 64 & 3.30 & 123 & 6.34 & 0.57 & 0.43 & 0.76 \\
\hline
\end{tabular}




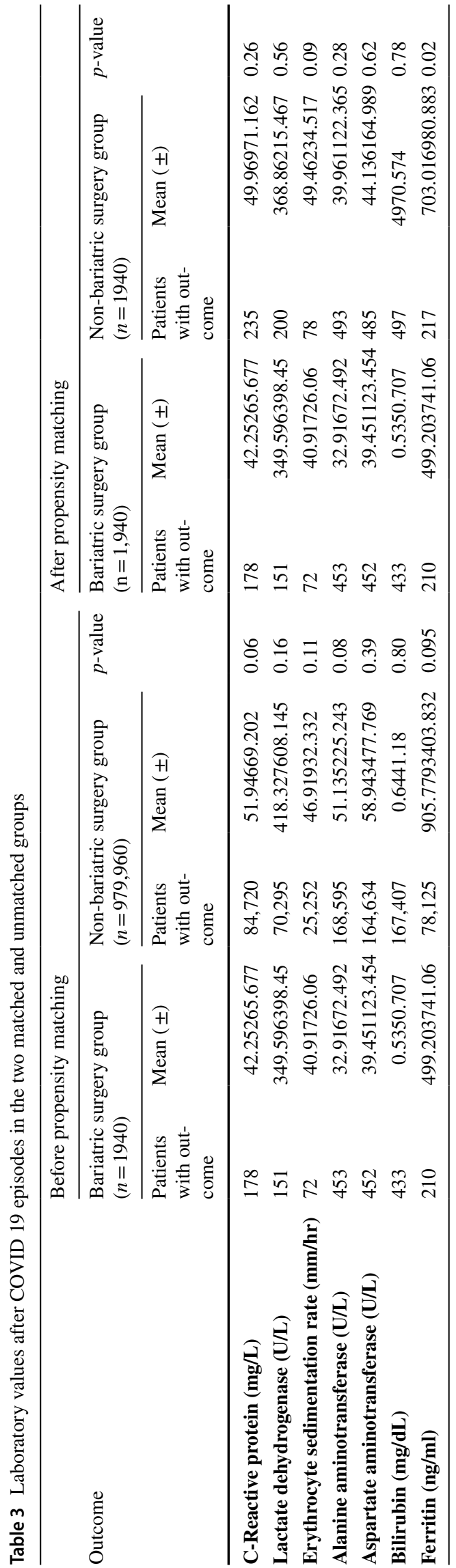

the study cohorts. Log rank tests revealed worse survival in both matched and unmatched analysis in the non-BS cohort compared to the BS group (log rank $p$ values $<0.01$ for all analyses). Kaplan-Maier survival curves are depicted in Supplementary Fig. 2a-d.

\section{Outcomes in RYGB and Sleeve Gastrectomy Subgroups}

Subgroup analysis showed no difference in outcomes in both unmatched and matched analyses. Characteristics of the subgroups are detailed in Supplementary Table 2 and clinical outcomes in both subgroups are compared in Table 4.

\section{Sensitivity Analysis}

On sensitivity analysis comparing BS group with BS qualifying cohort, lower risk of death and composite outcome was noted in the bariatric surgery cohort in unmatched analysis as shown in Supplementary Table 3. Furthermore, matching for demographic variables only, as well as a secondmatched analysis incorporating all covariates mentioned in the methods section also revealed a lower rate of mortality, mechanical ventilation, need for critical care, and composite outcome in the bariatric surgery cohort when compared to patients who qualified for bariatric surgery, as detailed in Supplementary Table 4.

\section{Discussion}

Bariatric surgery is associated with multiple health benefits in patients with obesity. It has previously been shown to improve diabetes, cardiovascular health, as well as general wellbeing among patients with obesity [13, 14]. Patients with severe obesity have been found to be at increased risk of hospitalization and mortality from COVID-19 disease [11]. Our large research network analysis found better outcomes of COVID-19 disease in patients with a history of bariatric surgery. These patients were at lower risk of mortality and need for mechanical ventilation after COVID-19 diagnosis. Even with the presence of a significant burden of co-morbid diseases, patients with prior bariatric surgery had a lower risk of severe COVID-19.

Previously, a study from Cleveland Clinic Ohio compared COVID-19 illness of 33 patients with prior history of bariatric surgery with obese patients with a BMI greater than $40 \mathrm{~kg} / \mathrm{m}^{2}$ and noted a lower rate of hospitalization in the bariatric surgery group [9]. Subsequently, an inpatient administrative data retrospective study from France has indicated a lower risk of mechanical ventilation or mortality in patients with a history of bariatric surgery when compared to hospitalized patients with obesity [15]. However, these studies 
Table 4 Outcomes in RYGB and SG cohorts before and after propensity score matching

\begin{tabular}{|c|c|c|c|c|c|c|c|}
\hline \multicolumn{8}{|c|}{ Before propensity score matching } \\
\hline Outcome & RYGB group & Percentage & SG group & Percentage & Risk ratio & $95 \%$ CI lower & $95 \%$ CI upper \\
\hline Mortality within 30 days & - & - & - & - & - & - & - \\
\hline Inpatient services & 90 & 10.15 & 97 & 10.19 & 0.99 & 0.76 & 1.31 \\
\hline Critical care & 20 & 2.26 & 24 & 2.52 & 0.89 & 0.50 & 1.61 \\
\hline Mechanical ventilation & - & - & - & - & - & - & - \\
\hline 30-day composite outcome & - & - & - & - & - & - & - \\
\hline Acute kidney injury & 24 & 2.71 & 36 & 3.78 & 0.72 & 0.43 & 1.19 \\
\hline \multicolumn{8}{|c|}{ After propensity score matching } \\
\hline Outcome & RYGB group & Percentage & SG group & Percentage & Risk ratio & $95 \%$ CI lower & $95 \%$ CI upper \\
\hline Mortality within 30 days & - & - & - & - & - & - & - \\
\hline Inpatient services & 73 & 10.47 & 60 & 8.61 & 1.22 & 0.88 & 1.68 \\
\hline Critical care & 14 & 2.01 & 13 & 1.87 & 1.08 & 0.51 & 2.27 \\
\hline Mechanical ventilation & - & - & - & - & - & - & - \\
\hline 30-day composite outcome & - & - & - & - & - & - & - \\
\hline Acute kidney injury & 19 & 2.73 & 21 & 3.01 & 0.91 & 0.49 & 1.67 \\
\hline
\end{tabular}

(-) TriNetX obfuscates patient counts when aggregate count $\leq 10$ to safeguard protected health information

were limited by small sample size, inclusion of mostly hospitalized patients and composition of control groups. Our matched analysis of a large cohort confirms and expands the understanding of COVID-19 disease in this population. It can be deduced from our data that bariatric surgery does not pose a risk at the time of COVID-19 pandemic, and thus these procedures do not warrant a delay during this time. Better outcomes of COVID-19 appear to be another benefit of bariatric surgery in this population.

Perhaps the most extraordinary association, although indirect, that can be drawn from our data is the protective effect of prior bariatric surgery against a severe disease course of COVID-19, and this may be independent of the weight loss associated with these procedures and their effect on other co-morbid diseases. We noted a decreased risk of severe outcomes in the bariatric surgery group compared to patients without a history of bariatric surgery in our crude unmatched analysis with a control group of participants with lower average BMI and much lower prevalence of diseases including chronic kidney disease, hypertension, diabetes, ischemic heart disease, and heart failure. Therefore, it can be inferred that the protective effect cannot all be attributed to a decrease in metabolic risk factors and BMI that may be associated with a history of bariatric procedures. Furthermore, robust sensitivity analysis controlling for metabolic parameters showed improved outcomes in the bariatric surgery cohort. One possible explanation for this observation is that ACE- 2 is substantially expressed in gastric mucosa, and therefore a bypass or exclusion of the stomach can be hypothesized to lead to a decrease in the inoculant dose of COVID-19. This same mechanism may explain the low incidence of COVID-19 previously noted among patients with a history of bariatric surgery [16]. However, this hypothesis is purely speculative in nature and needs to be further studied. Bariatric surgery patients are a motivated and mostly compliant cohort as they are actively engaged in a program to improve their health. This increased health awareness and behavior modification may contribute to the risk of contracting COVID-19; however, this should not explain improved disease outcomes once infected. It is also possible that bariatric surgery patients have better access to healthcare than the general population. During the current phase of the COVID-19 pandemic and going forward, vaccination status will have an enormous impact on the severity and outcomes of COVID-19. In our study, a very small proportion of patients in both the BS and non-BS cohort were vaccinated because of the unavailability of vaccines to the general population during the study period.

The main strengths of our study include the large and diverse cohort captured by our analysis and the robust control we were able to achieve for confounders. Multiple sensitivity analysis also lends strength to our findings. We included both inpatient and outpatient populations to give a more "real-world" analysis of COVID-19 outcomes after bariatric surgery. The main limitations include biases inherent to most studies conducted on electronic health record data. Care was taken to limit outcomes to those that are less likely to suffer from inaccuracies in EHR documentation, and any residual "pollution" should represent a minor challenge to our conclusions.

In conclusion, in our propensity-matched analysis of a large cohort, we have found that a history of bariatric surgery is associated with a reduced risk of poor outcomes in patients with COVID-19. 
Supplementary Information The online version contains supplementary material available at https://doi.org/10.1007/s11695-021-05803-1.

\section{Declarations}

Conflict of Interest The authors declare no competing interests.

\section{References}

1. Muller L, Gorter K, Hak E, et al. Increased risk of common infections in patients with type 1 and type 2 diabetes mellitus. 2005;41(3):281-8.

2. Simonnet A, Chetboun M, Poissy J, et al. High prevalence of obesity in severe acute respiratory syndrome coronavirus-2 (SARS-CoV-2) requiring invasive mechanical ventilation. 2020;28(7):1195-9.

3. Lighter J, Phillips M, Hochman S, et al. Obesity in patients younger than 60 years is a risk factor for Covid-19 hospital admission. 2020;71(15):896-7.

4. Popkin BM, Du S, Green WD, Beck MA, Algaith T, Herbst $\mathrm{CH}$, et al. Individuals with obesity and COVID-19: a global perspective on the epidemiology and biological relationships. 2020;21(11):e13128.

5. Albashir AADJCM. The potential impacts of obesity on COVID-19. 2020;20(4):e109.

6. Sattar N, McInnes IB, McMurray JJJC. Obesity is a risk factor for severe COVID-19 infection: multiple potential mechanisms. 2020;142(1):4-6.

7. Campos GM, Khoraki J, Browning MG, et al. Changes in utilization of bariatric surgery in the United States from 1993 to 2016. 2020;271(2):201-9.

8. Xu J, Chu M, Zhong F, et al. Digestive symptoms of COVID-19 and expression of ACE2 in digestive tract organs. 2020;6(1):1-8.
9. Aminian A, Fathalizadeh A, Tu C, et al. Association of prior metabolic and bariatric surgery with severity of coronavirus disease 2019 (COVID-19) in patients with obesity. 2021;17(1):208-14.

10. Aminian A, Tu CJOs. Association of bariatric surgery with clinical outcomes of SARS-CoV-2 Infection: a systematic review and meta-analysis in the initial phase of COVID-19 pandemic. 2021:1-7.

11. Singh S, Bilal M, Pakhchanian $H$, et al. Impact of obesity on outcomes of patients with coronavirus disease 2019 in the United States: a multicenter electronic health records network study. Gastroenterology. 2020;159(6):2221-2225.e6. https://doi.org/10. 1053/j.gastro.2020.08.028.

12. Singh S, Khan A, Chowdhry M, et al. Risk of severe coronavirus disease 2019 in patients with inflammatory bowel disease in the United States: a multicenter research network study. Gastroenterology. 2020;159(4):1575-1578.e4. https://doi.org/10.1053/j. gastro.2020.06.003.

13. Doumouras AG, Wong JA, Paterson JM, et al. Bariatric surgery and cardiovascular outcomes in patients with obesity and cardiovascular disease: a population-based retrospective cohort study. Circulation. 2021;143(15):1468-80. https://doi.org/10.1161/ CIRCULATIONAHA.120.052386.

14. Schauer PR, Bhatt DL, Kirwan JP, et al. STAMPEDE Investigators Bariatric Surgery versus intensive medical therapy for diabetes 5-year outcomes. N Engl J Med. 2017;376(7):641-51. https://doi. org/10.1056/NEJMoa1600869.

15. Iannelli A, Bouam S, Schneck A-S, et al. The impact of previous history of bariatric surgery on outcome of COVID-19. A Nationwide Medico-Administrative French Study. 2020:1-9.

16. Romero-Velez G, Pereira X, Skendelas JP, et al. Diagnosis of COVID-19 and the bariatric surgery population: a single center experience. 2021:1-6.

Publisher's Note Springer Nature remains neutral with regard to jurisdictional claims in published maps and institutional affiliations.

\section{Authors and Affiliations}

\section{Yousaf Bashir Hadi ${ }^{1} \cdot$ Rupinder Mann $^{2} \cdot$ Amir Humza Sohail $^{3} \cdot$ Miles Graves $^{4} \cdot$ Nova Szoka $^{5} \cdot$ Salim Abunnaja ${ }^{5}$. Lawrence E. Tabone ${ }^{5}$. Shyam Thakkar ${ }^{1}$. Shailendra Singh ${ }^{1}$}

1 Section of Gastroenterology and Hepatology, West Virginia University School of Medicine, 1 Medical Center Dr \# 2283, Morgantown, WV 26506, USA

2 Department of Internal Medicine, Saint Agnes Medical Center, 1303 E Herndon Ave, Fresno, CA 93720, USA

3 Department of Surgery, NYU Langone Hospital-Long Island, 222 Station Plaza North, Fifth Floor, Suite 510, Mineola, NY 11501, USA
4 Department of Medicine, West Virginia University School of Medicine, 1 Medical Center Dr \# 2283, Morgantown, WV 26506, USA

5 Department of Surgery, West Virginia University School of Medicine, 1 Medical Center Dr \# 2283, Morgantown, WV 26506, USA 